Informal Logic

X.3, Fall 1988

\title{
reply
}

\section{Logical and Extralogical Constants}

\section{ROGER SMOOK University of Guelph}

Some logicians propose to define logical consequence on the basis of a distinction between 'logical' and 'extralogical' constants. In the first and second parts of this paper I will criticize two recent examples of this approach due respectively to Rolf George ${ }^{1}$ and David Hitchcock ${ }^{2},{ }^{3}$. In the third part I will distinguish in my own way between logical and extralogical constants. The distinction, as understood by me, presupposes logical consequence hence cannot, on pain of vicious circularity, be appealed to in defining it. However the distinction can be enlisted without circularity in the definition of 'formal' logical consequence. My approach is motivated by the belief that for purposes of ordinary, everyday logic (what is usually called informal logic or critical thinking) there is no other useful or relevant way of drawing the distinction.

\section{I}

George defines logical consequence as follows:

The sentence $\mathrm{X}$ follows logically from the set of sentences $K$ if, and only if, every uniform substitution upon all extralogical constants of $\mathrm{K}$ and $\mathrm{X}$ which turns all the sentences of $\mathrm{K}$ into true sentences also turns $\mathrm{X}$ into a true sentence. ${ }^{4}$

Unfortunately this is not a satisfactory definition of logical consequence. For one thing George's definition could at best serve to characterize formal logical consequence. It fails to embrace such an example of logical consequence as the following: 'Yesterday was Sunday, therefore today is Monday. " 5

Another problem is that intuitively we want to conceive an extralogical constant in such manner that some extralogical constants can have parts which are in turn extralogical constants. The intuitive extralogical sentential constant 'Sam is small and Tom is tall' contains the extralogical constants 'Sam is small' and 'Tom is tall' as parts. But from this standpoint George's reference to all extralogical constants is nonsense. His definition cannot be meaningful construed so as to countenance independent substitution for both atomic and molecular extralogical constants.

The worst defect of the definiton is that George bases it upon a wholly unclarified use of the term 'extralogical constant'. If the definition is to serve a useful purpose we must have a sure criterion for which expressions are extralogical constants. If we are free simply to stipulate which expressions are extralogical constants, then, absurdly, any argument having a true conclusion can be considered logically valid on the basis of the definition. It suffices to stipulate that no expression in the argument is an extra-logical constant.

As Hitchcock observes, George seems to base his idea of an extralogical constant upon certain formal languages-viz. . sentential logic and first order predicate calculus. ${ }^{6}$ However if this is so. then the 
definition rests upon nothing more solid than a counter-stipulation. Certain constants of certain rigidly structured languages or supposed ordinary-language equivalents thereof are to count as logical constants. Constants not falling into this favoured group are to count as extralogical. But this approach eschews any real philosophical 'coming to grips' with what a logical or nonlogical constant is. Moreover it gives unacceptable results. George is led to say that the following argument is not logically valid-i.e., that the conclusion is not a logical consequence of the explicitly stated premisses: 'Al is older than Bill, Bill is older than Charlie, therefore $\mathrm{Al}$ is older than Charlie'. But this is intuitively wrong. I think we can 'see' that the conclusion does follow and moreover that it follows in a purely formal way in the sense that we could substitute 'Alphonse' for 'Al', 'Boris' for 'Bill' and 'Chlodwig' for 'Charlie' and still preserve the relation of logical consequence. Why does George think the conclusion does not follow? Evidently he construes 'is older than' as an extralogical constant since, according to him, the conclusion follows only if we add the extra premiss: 'For all $x$, $y$ and $z$ : if $x$ is older than $y$ and $y$ is older than $z$, then $x$ is older than $z$.' However George's own definition of logical consequence bears out the intuitive view that the extra premiss is not needed provided only we construe 'is older than' as a logical, rather than extralogical, constant. But it is not yet clear on what theoretical basis we should do so.

\section{II}

The following definition of formal logical consequence of David Hitchcock is an improvement over George's:

An argument is formally deductively valid [i.e., its conclusion is a formal logical consequence of its premisses] if and only if no uniform substitution on the argument's atomic content ex- pressions produces an argument with true premisses and a false conclusion. ${ }^{7}$

For one thing, Hitchcock is aware of the fact that a definition of this sort can characterize at best formal logical consequence but not logical consequence simpliciter. For another thing, Hitchcock avoids George's fault of seeming to countenance the absurdity of independent substitutions for both atomic and molecular expressions.

Evidently Hitchcock uses 'content expression' more or less equivalently with George's 'extralogical constant'. However Hitchcock wishes his definition to apply to natural languages. He therefore offers the following characterization of content expression:

Such expressions, the categorematic terms of medieval logic, can be regarded as referring to or otherwise signifying actual or possible features of the universe: entities, qualities, occurrent states, dispositions, events, relationships, times, places, facts and so forth. Natural languages thus have a built-in categorical scheme, which could in principle be made explicit... Content expressions can be defined in terms of this apparent categorical scheme as expressions which in the context of their utterance can be regarded as referring to or otherwise signifying an item in a category. ${ }^{8}$

The following points made by Hitchcock are also of importance for understanding his definition:

(I originally introduced the term "category" for categories of items; by extension, one can speak of the category to which belongs an expression signifying an item in a category.) ...Let us define substitution on a content expression as "replacement of that content expression by a content expression in the same category". (We allow as a degenerate case substitution of a content expression by itself.) Further, by uniform substitution on a content expression let us mean "replacement of all occurrences of a content expression by the same content expression, one in the same category as the original". (It is to be understood that the expression has the same meaning at all occur- 
rences; where the expression has different meanings at different occurrences, we treat these as occurrences of different content expressions.) ${ }^{9}$

I agree with Hitchcock that "natural languages have a built-in categorical scheme, which could in principle be made explicit." (I will in fact base my own position, to be developed in Part III, upon this assumption.) Unfortunately, however, it is easy to show that his definition can not do the job it is supposed to do.

The English words 'is older than' clearly stand for a relationship, hence for a categorical feature of the universe, as understood by Hitchcock. Therefore 'is older than' must constitute for Hitchcock a content expression. Further, since it can't be broken down into smaller content expressions, it must count for him as atomic. (In case one is disturbed by the copula, I could reformulate my argument in terms of just 'older than'-i.e., I needn't insist that the 'is' is part of the content expression.) Observe now that Hitchcock's definition leads to construal of the following-already argued to be formally valid - as formally invalid: ' $\mathrm{Al}$ is older than Bill, Bill is older than Charlie, therefore $\mathrm{Al}$ is older than Charlie.'

Suppose Alphonse is the father of Boris, Boris the father of Chlodwig and make the substitutions of 'Alphonse' for 'Al', 'Boris' for 'Bill', 'Chlodwig' for 'Charlie' and 'is the father of' for 'is older than'. (Note that 'is the father of' and 'is older than' refer to items of the same category and are hence intersubstitutable content expressions.) Clearly the premisses of the new argument are true and its conclusion false. This suffices to show the unsatisfactoriness of Hitchcock's definition of formal validity.

Hitchcock defines logical consequence simpliciter in terms of formal logical consequence. Thus he says, "We can define a deductively valid argument as an argument which is either formally deductively valid or can be made so by the addition of one or more definitionally true premisses. "10
This definition is unsatisfactory, if for no other reason, because it is based upon a notion of formal deductive validity which is unsatisfactory.

Both George and Hitchcock assume that logical consequence can be defined (and defined only) on the basis of an antecedent distinction between logical and extralogical constants. Perhaps there is some sense of 'logical consequence' (as well as some way of drawing the distinction) for which this is true. However the assumption seems questionable in regard to logical consequence in the sense presupposed by ordinary reasoning. ${ }^{11}$

\section{III}

Let me now distinguish in my own way between logical and extralogical constants. I begin with the following definition of extralogical constant:

A non-empty set of content expressions $\mathrm{W}$ is a set of extralogical constants relative to a particular argument $\mathrm{A}$ if, and only if, all members of $\mathrm{W}$ occur in A and every uniform substitution upon all members of $\mathrm{W}$ in $\mathrm{A}$ results in an argument whose conclusion is a logical consequence of its premisses. ${ }^{12}$

Note the salient points: (1) that an expression is an extralogical constant only relatively speaking-i.e., in the context of a particular argument; (2) that, far from its being the case that we can use the notion of an extralogical constant to define logical consequence, the notion itself cannot be defined except in terms of logical consequence. (Can logical consequence be defined at all? I think not. I am in agreement with those philosophers who think that only a more or less circular definition can be provided-i.e., that logical consequence can be 'defined' only in terms of such cognate notions as logical necessity, logical possibility, logical consistency, etc.) 
In terms of my definition of extralogical constant, formal logical consequence can now be defined as follows:

An argument $\mathrm{A}$ is formally valid (its conclusion is a formal logical consequence of its premisses) if, and only if, there exists a set of extralogical constants relative to $\mathrm{A}$.

Other senses of 'formal logical consequence' are, of course, possible. It seems to me, however, that this sense, and only this, is clearly relevant to ordinary logic. ${ }^{13}$

What is a logical constant? It seems to me that in the primary instance a logical constant has to be conceived as the whole framework of an argument considered in abstraction from some set which is a set of extralogical constants relative to it. True we sometimes say of some isolated expression-e.g., 'or'-that it is a logical constant. But this is due in the end to the fact that the expression in some contexts just is-or, at least, approximates very nearly to-such a framework. Consider the argument 'Tom is tall, therefore Tom is tall or Sam is short.' If we consider this is abstraction from the extralogical constants 'Tom is tall' and 'Sam is short' we get 'or' or more precisely, perhaps, we get something like " , therefore or ..... 14

The same expression can be either extralogical or logical depending upon context. In the context, of the argument, 'Al is older than Bill, Bill is older than Charlie, therefore $\mathrm{Al}$ is older than Charlie', the expression 'is older than' is a logical constant. ${ }^{15}$ But in the context of the argument, 'John is identical with the captain of the basketball team, John is older than Ron, therefore the captain of the basketball team is older than Ron', it is an extralogical constant. In the first argument we may substitute ad libitum any names of individuals for 'Al', 'Bill' and 'Charlie' without jeopardizing logical validity. But we are not free to substitute any arbitrary expression referring to a relation for "is older than'. In the second argument we can substitute freely, not only for 'John' and 'Ron' but also for 'is older than'. ${ }^{16}$

\section{Notes}

1 Rolf George, 'Enthymematic Consequence', American Philosophical Quarterly 9 (1972), 113-116. I have added the word 'uniform'. By a uniform substitution upon an extralogical constant is meant replacement of all occurrences of that extralogical constant by the same extralogical constant (which, in the normal case, is different from the original). Also I have corrected 'all the sentence of $\mathrm{K}$ ' to read 'all the sentences of $\mathrm{K}^{\prime}$.

2 David Hitchcock, 'Enthymematic Arguments', Informal Logic VII (1985), 83-97.

3 I believe that the theory of enthymemes set forth by George and elaborated by Hitchcock is vitiated by mistaken ideas about logical vs. extralogical constants and about logical consequence. Here however I exclude enthymemes from the scope of my critique.

4 George, p. 113.

5 The example is Hitchcock's. See Hitchcock, p. 85.

6 Hitchcock, p. 83.

7 Hitchcock, p. 85.

8 Hitchcock, p. 84.

9 Hitcheock, p. 84.

${ }^{10}$ Hitchcock, p. 86.

${ }^{11}$ See footnote 13.

12 I use 'content expression' and 'uniform substitution' in the same manner as Hitchcock. See, in this regard, his previously cited remarks. There is however a problem due to the fact that one extralogical constant could be contained within another larger extralogical constant at a 
particular occurrence. In order to deal with this possibility a somewhat more complicated definition is required. Let me say that a particular member of the expression-set $\mathrm{W}$ has an independent occurrence in A (relative to W) if, and only if, it is not contained within some other member of $\mathrm{W}$ at that occurrence. Further let us understand by a W-restricted uniform substitution upon a content expression in A a replacement of all and only independent occurrences of that content expression in A by the same content expression (one in the same category as the original). I am now in a position to formulate what I believe is a satisfactory definition:

A non-empty set of content expressions $W$ is a set of extralogical constants relative to a particular argument $A$ if, and only if, all members of $W$ have some independent occurrences in A and every W-restricted uniform substitution upon all members of $\mathrm{W}$ in A results in an argument whose conclusion is a logical consequence of its premisses.

Note how this definition allows us to conclude that 'the brother of Paul', 'Paul' and 'the sister of Paul' are the members of a set of extralogical constants relative to the argument: 'The brother of Paul is taller than Paul, Paul is taller than the sister of Paul, therefore the brother of Paul is taller than the sister of Paul.'

${ }^{13}$ I do not wish to exagerate the importance of this particular definition of formal logical consequence. What is essentially the same notion can be captured in another, perhaps simpler way. Thus the usual procedure is to introduce the con- cept of a 'valid argument form'. A formally valid argument is then definable as an instance of a valid argument form. I have no objection to this procedure. But I think it is of interest that formal logical consequence can be defined without resort to the concept of a valid argument form.

14 According to this explication a logical constant is essentially the same as a "valid argument form'. Since logical constant and extralogical constant are correlative notions either one of them is easily definable in terms of the other.

${ }^{15}$ More precisely, 'is older than' is the sole constant (apart form 'therefore') occurring in the valid argument form '_ is older than .....,.... is older than $\& \& \& \& \&$, therefore is older than $\& \& \& \& \&$ '.

16 There are innumerable ways in which the distinction between logical and extralogical constants could be drawn. Two interesting approaches are those of Christopher Peacocke and Ian Hacking respectively. See Peacocke, 'What Is a Logical Constant?', Journal of Philosophy, LXXVI (1976), 221-240 and Hacking, 'What Is Logic?', Journal of Philosophy, LXXVI (1979), 285-319. Unfortunately neither approach is germane to the project of defining logical consequence or formal logical consequence, so far as these notions pertain to ordinary (informal) logic.

Roger Smook, Department of Philosophy, University of Guelph, Guelph, Ontario, NIG $2 W 1$. 